\title{
Inside 50,000 living rooms: an assessment of global residential ornamentation using transfer learning
}

\author{
Xi Liu', Clio Andris ${ }^{1 *}$, Zixuan Huang ${ }^{2}$ and Sohrab Rahimi ${ }^{3}$
}

\author{
"Correspondence: clio@psu.edu \\ 'Department of Geography, The \\ Pennsylvania State University, \\ University Park, USA \\ Full list of author information is \\ available at the end of the article
}

\begin{abstract}
The global community decorates their homes based on personal decisions and contextual influences of their larger cultural and economic surroundings. The extent to which spatial patterns emerge in residential decoration practices has been traditionally difficult to ascertain due to the private nature of interior home spaces. Yet, measuring these patterns can reveal the presence of geographic culture hearths and/or globalization trends.

In this work, we collected over one million geolocated images of interior living spaces from a popular home rental website, Airbnb (http://airbnb.com), and used transfer learning techniques to automatically detect the presence of key stylistic objects: plants, books, decor, wall art and predominance of vibrant colors. We investigated patterns of home decor practices for 107 cities on six continents, and performed a deep dive into six major U.S. cities.

We found that world regions show statistically significant variation in decorative element prevalence, indicating differences in geographic cultural trends. At the U.S. neighborhood level, elements were only weakly spatially clustered and found to not correlate with socio-economic neighborhood variables such as income, unemployment rates, education attainment, residential property value, and racial diversity. These results may suggest that American residents in different socio-economic environments put similar effort into personalizing and caring for their homes. More broadly, our results represent a new view of worldwide human behavior and a new application of machine learning techniques to the exploration of cultural phenomena.
\end{abstract}

Keywords: Computer vision; Interior decor; Maps; Indoors; Homes; Spatial analysis

\section{Introduction}

Interior decoration and ornamentation is a key form of human self-expression and communication of values and ideals [1-3]. This is especially true in the home, where residents and owners have agency to personalize their surroundings and cultivate a space for themselves and visitors [4]. Configuring a domestic space has both internal and contextual (socio-cultural and geographic) components, which together reflect a need for privacy, interpersonal relationships, symbolization of the self, and aesthetic stimulation [5]. Although ornamentation is known as an indicator of social and economic status [6], today,

(c) The Author(s) 2019. This article is distributed under the terms of the Creative Commons Attribution 4.0 International License (http://creativecommons.org/licenses/by/4.0/), which permits unrestricted use, distribution, and reproduction in any medium, provided you give appropriate credit to the original author(s) and the source, provide a link to the Creative Commons license, and indicate if changes were made. 
large-scale manufacturing as well as a culture of re-sale and re-use has encouraged people of varying means to customize their living spaces with affordable choices [2] including affordable methods of painting, re-purposed materials, and old or free furnishings. Even wealthier homeowners have strayed from choosing professional decorators, [7] instead embracing a growing trend of do-it-yourself (DIY) culture. While there may be unequal access to interior decor elements and the ability to purchase these goods in all global regions, globalization and the "IKEAficiation" of furniture has penetrated urban and rural regions in the developed and developing world alike [8]. If similar elements are available around the world, there may be an increasing convergence between international cultural decorative behaviors that reveal an underlying similarity in decorative preferences regardless of locality.

Given the increasing accessibility and globalizing influence of material decor, a key question for the modern assessment of residential life is whether interior living spaces exhibit similar decorative properties, or if these properties differ by geographic region. Accordingly, the research question addressed in this work is: To what extent is residential ornamentation behavior localized into spatial clusters and regions? Our research objectives are to successfully detect ornamentation evidence from a large set of global interior living rooms and to discover the extent of statistical geographic variation in interior ornamentation choices across global regions and across inter-city neighborhoods. Our null hypothesis is that each ornamentation element is distributed randomly across geographic space, and alternative hypothesis is that there are distinct geographic trends inherent to the presence of these elements.

Here, we examine a set of global cities using images from a popular home rental site, Airbnb (http://airbnb.com). Airbnb allows members to list their personal homes as an alternative to hotels for visitors, and provides interiors images of the spaces for rent in their postings. Airbnb was chosen for its international penetration, corporate validity and temporal online persistence, as well as its ability to represent thoughtfully-photographed global residential interiors, i.e. everyday homes. This site offers over five million listings in 191 countries and 81,000 locales. The listed rentals are uniquely created and furnished by individuals and families (although exceptions exist). While they can be decorated to entice consumers [9], the interiors also have an added personal dimension of individual decor and style choices. We originally collected over one million listing images and analyzed over 130,000 images representing about 54,000 living rooms from 107 global cities in 65 countries over six continents, and in detail in six U.S. cities (Chicago, Houston, Los Angeles, New York, Philadelphia and Washington, D.C.). We specifically chose listings where renters rented a bedroom in a unit or home, i.e. not a hostel-style room or the whole home/unit, in order to capture homes where the owner may live. While it is not guaranteed that the owner also lives on site, this check increases the likelihood that the owner lives in the house and ostensibly decorated the common spaces [10].

We codify ornamentation behaviors as the presence of indoor plants, books, wall art, general decor, and vibrant paint colors within living rooms, as they met two criteria. First, these "small" (movable, lightweight) choices communicate a personal decision to decorate (unlike a wall-to-wall carpet from a rental unit, built-in lighting, or architectural design). Second, these objects were detected with high success rates in the machine learning process. Third, these elements have been previously listed in former research on interior de- 
sign theory via qualitative interior photograph analysis $[5,11]$. In the following sections, we describe prior research, our dataset, processing, methods and results.

\section{Related work}

\subsection{Conceptually-related work}

Our research question is examined through an exploratory data science lens that takes advantage of a new trove of online geolocated images. Today, the quantitative analysis of large datasets of user-generated image content is a burgeoning research task. Image analysis techniques often detect objects and report descriptive statistics, and more recently have advanced toward more 'intelligent' tasks. Regarding interiors, machine learning methods have been used to recognize types of "style" from large collections of photographs of keyword-tagged furniture [12] and art using a set of classified Wikipaintings images [13]. Resulting classes include "vintage", "rustic", "baroque", and "modern", which help characterize the design style of an interior quickly and efficiently.

At the scale of the interior room, both commercial and residential interiors can be analyzed with machine learning methods. Interior images from Zillow.com and Houzz.com have been classified by levels of "luxury" to improve estimates of home valuation and sale price prediction, [14]. A similar study used machine learning methods on photographs of restaurant interiors uploaded by Yelp.com users in order to predict restaurant success [15].

When photographs are geolocated, different image qualities can be mapped to neighborhoods and cities. For example, [16] detected different ambiance from crowdsourced Foursquare images and found that cities like Barcelona had 'artsy' photos, while Paris had more 'romantic' scenes. Objects within the city are also studied. Object detection methods have been used to detect the makes and models of cars from Google Street View images and associate car type with the surrounding neighborhood demographics using GIS analysis [17]. Next, the locations of different types of street art have been detected from geolocated Instagram photos [18] to produce maps that allow visitors and residents to locate and appreciate the different artistic offerings local cities. Lastly, even the presence of environmental features-the locations of flowers and snow-have been mapped using geolocated Flickr photos [19].

At the intersection of interior image analysis and the spatial analysis of processed image features, two recent studies detected color and ambiance from online interior rental photos [20,21], finding differences in interior decor across continents using two nations, and 10 cities, respectively. Our study's research design is modeled after these examples.

\subsection{Background on geographic and cultural variation of interior elements}

The study of geographic variation in residential design has overwhelmingly focused on exterior architecture, building materials and cultural use of space in homes [22]. However, there is some indication that decorative emblems and styles differ across geographic spaces, regions and countries. For instance, it was found that traditional French cultures value ornateness in their choice of decor, while Italians prefer objects with functional purposes [5]. In terms of our chosen elements, various scholars have examined the presence of books, plants, the abundance of wall art and ornaments [5], and the presence of predominant colors across global cities through photographs [11]. 


\subsubsection{Elements}

The presence of books in the home has historically reflected the pursuit and investment in knowledge [23]. More specifically, the size of the home library is correlated with, and is used to define, a household's "scholarly culture" [23-25] via [26]. The prevalence of books in interiors has been linked to education and literacy rates, as found by a survey conducted in 43 nations [27] wherein the fewest residential books appeared in Macedonia, Brazil, Indonesia and Romania and the most reading material in Hungary, Czech Republic, Austria, and Germany [26].

The practice of keeping indoor plants has been studied as an important expression of organic decoration i.e. "biophilia" [28], which psychologically brings inhabitants closer to nature. Interior plants have been studied for their health and workplace benefits. The presence of indoor plants has been said to create an environment that promotes wellness and holistic health [29], and indoor foliage has been shown to improve health and reduce stress in hospital patients [30]. In another study, after the introduction of office flora, worker participants were more productive and less stressed [31]. It has also been shown that windows overlooking greenery produce the same stress-reducing effects as having indoor plants [32]. However, it is unclear specifically where, geographically, plant-rearing is a common practice

The study of global differences of art and decor within homes has been approached through the study of material culture, which stresses the decorative or functional utility of items [33] or object symbolism and anthropological practices related to objects (such as cooking) [34]. Fewer studies have delineated the geographic presence of a particular decorative element. Exceptions include the study of transferability of residential items through cultural diffusion. For instance, African-American Muslims in Philadelphia neighborhoods decorate their homes with melting-pot-like decor from the overseas Muslim world [35], while Indian immigrants in the United States define their interiors with symbolic elements of the religion and culture of their native nations [36].

\subsubsection{Color}

Cultural color perception and usage has been typically (and widely) studied alongside psychological and marketing topics of branding and affect (e.g. [37]) ranging from best practices for fast food restaurant walls to space station interior color [38]. In an international study of color preference, 21,060 subjects reported remarkably uniform preferences for, in order: blue, red, green, purple, orange, yellow [39], rendering lack of proof for racial or geographic difference in color preferences. More recently, a cross-cultural study including volunteers from multiple continents, detected similarities in color preferences (such as blue and black) and color meaning associations (such as red for aggression and power) [40]. Different cultures also report affective responses between colors: Afghan and Mysurur respondents reported the least affective differences between colors while Thai and Finnish subjects reported the highest affective differences between colors [41]. Similarly, [42] showed that American, British, Korean, and Japanese subjects described preferences for color elements including hue and chroma. Another color research study revealed that African American subjects preferred colors in a red-purple-black hue spectrum whereas white subjects tended to prefer greens and blue [40].

Color is also a key part of interior design, the topic of this work. Some interior color decisions are motivated by financial reasons. For example, international hotel chains have incorporated local cultural elements and their meanings in different geographic mar- 
kets to draw more guests [42]. Others are guided by government influence on culture: In the 1970s, the Singaporean Government warned citizens against using heavy colors and suggested more practical lighter colors which represented purported cleanliness ideals brought about by English colonialism, yet this rhetoric is not as common today [43].

There is also geographic variation in home color preferences. In a cross-cultural study of 12 sites, authors found that Greece dons colorful pastels; Scotland uses a deep, rich palette; India exhibits shades of blue; and Guatemala has a preference for teal [44]. Another study of geographic communities' use of color from natural and synthetic elements revealed a proliferation of blue and black in Europe, as well as red in Asia, among other trends [45]. In addition, exterior home colors in a contemporary Chinese city were found to lack a discernible statistical pattern but that color choices mixed throughout the city [46]. With these previous findings in mind, we next discuss the use of a large data corpus to assess urban interiors. Our approach extends the aforementioned research by using a high volume of cases to detect quantifiable, discrete objects and colors.

\section{Materials and methods}

\subsection{Data}

\subsubsection{Image retrieval}

We collected listing information and images from Airbnb's public API (https://www. airbnb.com/partner). Listing information included the home's location (longitude and latitude) and other properties such as price, amenities, and average customer rating out of five stars. Geographical coordinates were jittered and therefore slightly deviate from their exact location.

The data collection process was comprised of two main stages. We first collected the unique ids of listings located within the geographic area of interest. Due to API constraints, we collected listing ids by querying based on geographic identifiers, such as city name and zip code, which retrieved a maximum of 300 listing ids per name. We also queried by geographical bounding box, which returned a maximum of 50 listing ids per box. Once ids were retrieved, we then requested listing information and images through the API based on the retrieved listing ids.

Data and listing information were collected at both global and U.S. city levels in two waves (Oct. 2017 and Feb. 2018), respectively. At the global level, we collected data for 107 highly populated cities on six continents totaling over 38,000 listings (500,000 images). Cities were chosen based on population and availability of rentals. After image classification (described in the following section), and filtering for only living rooms, there remained 21,543 listings (49,000 images) for global analysis.

We also retrieved an oversample of data for six major U.S. cities, chosen by metropolitan area population: Chicago, IL; Houston, TX; Los Angeles, CA; New York, NY; Philadelphia, PA and Washington, DC. We first queried listing ids based on urban zip codes and then queried using $0.01^{\circ} \times 0.01^{\circ}$ bounding boxes across the city boundaries in order to improve geographic coverage. For the six cities, we collected over 50,000 listings (600,000 images). After image classification, and filtering for only living rooms, there remained 32,389 listings (81,000 images) for city-level analysis.

\subsubsection{Census data}

For each U.S. city, we characterized the neighborhoods of geolocated households using socioeconomic factors retrieved from the 2016 American Community Survey (ACS), in- 
cluding median household income, unemployment rate, percent of residents with bachelor's degrees, and median house value. We also computed an entropy-based racial diversity measure defined by the classic information entropy equation: $H(X)=-\sum_{i=1}^{n} p\left(x_{i}\right) \log p\left(x_{i}\right)$, where $p\left(x_{i}\right)$ is the proportion of a certain race/ethnicity (Asian, Black, Hispanic, White, and Other) within a tract.

\subsection{Methodology}

In this section, we introduce methods for retrieving living room images, analyzing ornamental elements and colors, and the statistical models used in the study. The filtering of living rooms from all images and the analysis of ornamental elements were conducted as image classification and object detection tasks, respectively (Fig. 1). We applied transfer learning techniques to customize the deep learning tools in computer vision to complete these tasks. Transfer learning, a technique commonly used in computer vision applications, refers to retraining a small number of parameters in a pre-trained deep learning model with customized data, in order to leverage the knowledge that the model has learned from massive data for a specific task with only a small number of training data. The image classification and object detection models were implemented with TensorFlow [47].

\subsubsection{Labeling training data}

To classify room types from the listing images, we manually labeled 36,657 photos. A set of training images was first labeled by workers on Amazon Mechanical Turk and then checked by graduate and undergraduate student volunteers, who also labeled some images directly. We ensured that the training data covered images from different geographical regions. To maintain the highest possible classification performance and create a balance across image categories (instead of using a binary label of "living room" and "non-living room") we used eight categories: living room, bedroom, kitchen, bathroom, dining room, other indoor rooms, object, and not indoor room.

To train object detection models, we labeled the training data by defining a bounding box around each object in the image and labeling the box by object type. Plants and books

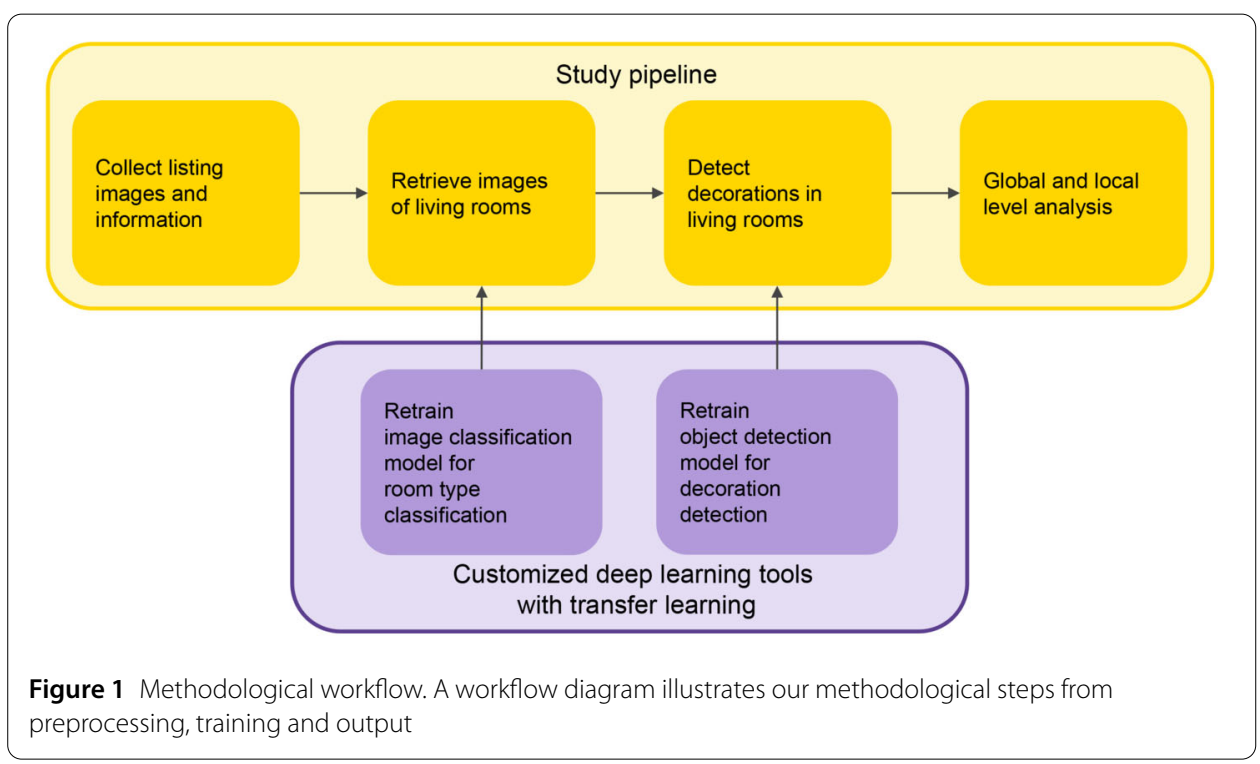


were detected using pre-trained models, so we only labeled images for training models to detect wall art and general decor. Using the Labellmg tool (https://github.com/tzutalin/ labelimg), student volunteers labeled 692 living room images as training data. For each image, the bounding box information for each wall art and decor object and their categories were recorded as XML files in PASCAL VOC format. Other potential false-positive objects in the images, such as televisions and windows, were also labeled to ensure that the re-trained model could distinguish between these similar objects (Fig. 2).

\subsubsection{Image classification}

We retrained the Inception V3 model [48] using the labeled data to classify listing images into the eight room type categories, and retrieved only living room images for the following analyses. The Inception V3 model was pre-trained with the ImageNet dataset [49] with $3.46 \%$ top-5 error rate. We retrained the last layer of the model (the fully connected layer) with $80 \%$ of the labeled photos, saving $20 \%$ for use as test data. The overall accuracy of our retrained model on the test set was $81.1 \%$. The living room category had a precision of $80.2 \%$ and recall of $75.3 \%$.

\subsubsection{Object detection}

The goal of the object detection task was to count the frequency of certain decoration elements in the living room images. For object detection of plants, wall arts, and decor, if a listing contained more than one living room image, we used the maximum number of each decoration element type to represent the number of the listing. For example, if listing A had two living room images, one with three pieces of wall art and the other with four, we would consider listing A to have four pieces of wall art.

We used and retrained the faster_rcnn_nas model in the TensorFlow Object Detection API [50] to detect decor in living room images. The model is based on Faster RCNN [51] and Neural Architecture Search (NAS) [52] methods. The model was pre-trained on the COCO dataset [53] and had the highest mean Average Precision (mAP) among all available models at the time of access. The pre-trained model was used directly to detect books and plants in each living room. We then retrained the model to detect wall art and decor with labeled images.

We hand-selected 100 living room images that contained various decorative elements and were not used in the training process to test the model's ability to detect the four ornamentation elements. We prioritized high precision over recall in our model since we combined the objection-detection results of multiple living room images for a single listing. This means that if an object was not detected in image A of a living room (a false negative), it could still be detected from image B of the same living room (producing a true positive). Moreover, when aggregating the object detection results for city-level or neighborhood-level analysis, a higher precision suggests that although the numbers may be smaller, they correctly reflect the real-world patterns. The precision and recall rates (Table 1) were both above 0.9 for wall art detection, while the remaining three elements had slightly lower recall rates. The elements that most frequently went undetected were books and decor, but the classifier rarely signaled elements that were not present (Table 1).

\subsubsection{Color detection}

We also detected predominant colors in each living room using HSV (hue, saturation, value) numbers to characterize colors found in the images. Hue represents the value on 

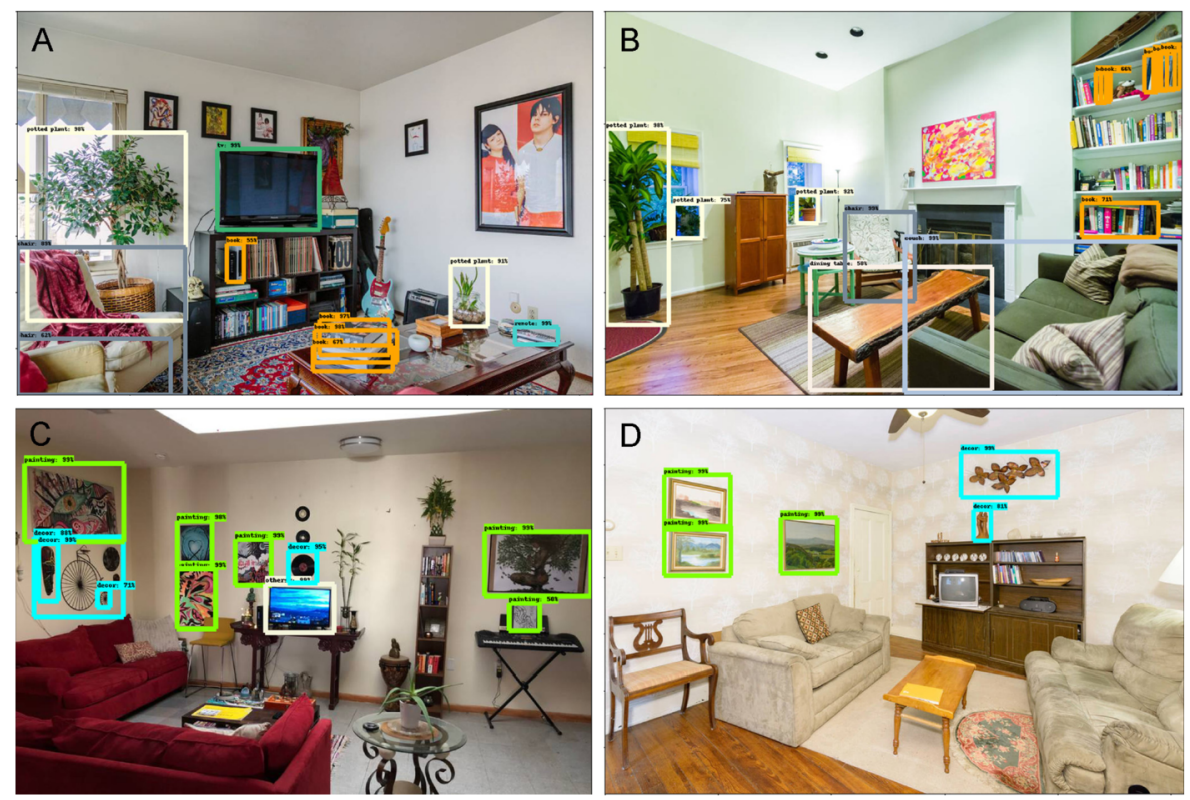

Figure 2 Object detection. Object detection examples for living room images. Ivory and orange bounding boxes in (a) and (b) are the model's results for plant and book identification, respectively. Green and blue bounding boxes in (c) and (d) are the model results for wall art and decor identification, respectively

the color spectrum, saturation describes a color's "purity" or how far it differs from gray, and value represents brightness along a white to black range [54]. To define "vibrant" colors, we set minimum thresholds of 0.50 saturation (range: $0-1$ ) and 0.75 value (range: $0-1$ ) and set no threshold for hue. These thresholds, while uncalibrated, ensured that no pastels, grays, or dark colors were included in the set of vibrant colors. We used the colorgram python package (https://github.com/obskyr/colorgram.py) to extract the 10 most dominant colors in each living room image (see Additional file 1 for more information). If one living room image contained a vibrant dominant color, we considered this listing to contain vibrant colors.

\subsubsection{Descriptive variable representation and statistical analysis}

Some living rooms contained many decorative elements, e.g. more than 40 small wall art pieces, which served as outliers in our analysis. Thus, we used the following numerical distinctions: plants, wall art and decor were each capped at 5 instances (so that if a living room has 10 plants, this is represented as 5). Books often appeared as piles or a shelf of books, and the model had difficulty distinguishing the exact number of books in the images. Thus, we considered the presence of books as a binary value, signifying the presence of any books (ranging from a single book to over 100 books), or a 0 for no detected books. Finally, the presence of at least one vibrant color in the top 10 major retrieved colors for an image was also presented as a binary variable. (See Additional file 1 for more information).

In the global analysis, we used Moran's I and ANOVA to detect spatial clustering and regional differences of image element preponderance, respectively. These tests were conducted using the city as the spatial unit of analysis. Moran's I was parameterized with a 4000-kilometer Haversine distance search radius and neighbor importance was weighted 
Table 1 Precision and recall for detecting the four decoration elements

\begin{tabular}{lll}
\hline Element type & Precision & Recall \\
\hline Plants & 0.984 & 0.709 \\
Books & 0.968 & 0.638 \\
Wall Art & 0.961 & 0.913 \\
Decor & 0.870 & 0.681 \\
\hline
\end{tabular}

using inverse-distance weighting. More information of the influence of parameters on results is listed in Additional file 1.

For U.S. cities, we used Moran's I to detect clusters of element concentrations in neighborhoods. Next, we examined whether the presence of certain living room elements correlated with the neighborhood's socio-economic indicators. We used linear regression (LR) and geographically weighted regression (GWR) to detect potential correlations: the dependent variables for our regressions were the detected elements (plants, books, etc.). The independent variables were socio-economic factors including median household income, unemployment rate, percent of residents with bachelor's degrees, median house value, and racial diversity retrieved from the U.S. Census.

Listings located in census tracts with incomplete census information (about 1.6\% of all the listings) were removed for the regression analysis. For LR, the values of interior elements were averaged by tract, and tracts with fewer than 5 rental listings were removed. For GWR, a regression equation was applied to each individual listing that included the socio-economic data of the listing's corresponding tract. As a result, each tract was given its own regression equation with unique variable parameters and $\mathrm{R}$ squared values. The optimal GWR Gaussian kernel bandwidth size (i.e. search radius) was calibrated to minimize cross-validation error using the spgwr package in $R$ (https://cran.r-project.org/web/packages/spgwr), and found to be 85 nearest neighbors.

\section{Results}

At the global level, an average of 201.34 private room listings with living room photos were detected in each city (min. 12 (Kinshasa, D.R.C.), max. 348 (Bogotá, Columbia)). The small numbers of detected residences in some cities were due to the limited availability of home offerings. (For residence count and resultant statistics for all 107 cities, see Additional file 2.)

\subsection{Global cities and regions analysis}

Most elements of interior design, including books, plants, wall art and color, were spatially clustered in the global city system. Their presence also differed significantly across regional culture hearths. In support of spatial clustering of cities with similar characteristics, a Moran's I test (Table 2), reported that books and wall art tended to have the most concentrated clustering patterns. The presence of books was most significantly clustered, and centered around Europe and the Americas (Fig. 3(b)). The presence of plants and vibrant colors were clustered less significantly, with plant concentrations in Northern Europe and China (Fig. 3(a)) and color concentrations in Japan, India and other locales (Fig. 3(e)). The presence of general decor in homes was not significantly clustered across global cities; cities exhibited high and low levels of decor with no observable spatial patterning. Across regions, the ANOVA F-test statistic revealed that the presence or absence of each element, except for decor, was more similar within regions than across regions (Table 2) and 
Table 2 Patterning of interior elements across global cities (Moran's I) and between regions (ANOVA)

\begin{tabular}{lllllll}
\hline Element & Moran's I & Moran's I Z-score & Moran's I P-value & ANOVA F Statistic & ANOVA P-Value & Pattern \\
\hline Plants & 0.254 & 5.326 & $<0.001$ & 5.344 & $<0.001$ & Clustered \\
Books & 0.298 & 6.209 & $<0.001$ & 5.094 & $<0.001$ & Clustered \\
Wall Art & 0.291 & 6.064 & $<0.001$ & 8.925 & $<0.001$ & Clustered \\
Decor & 0.104 & 2.288 & $<0.05$ & 1.463 & 0.181 & Random \\
Color & 0.132 & 2.877 & $<0.01$ & 2.797 & $<0.001$ & Clustered \\
\hline
\end{tabular}

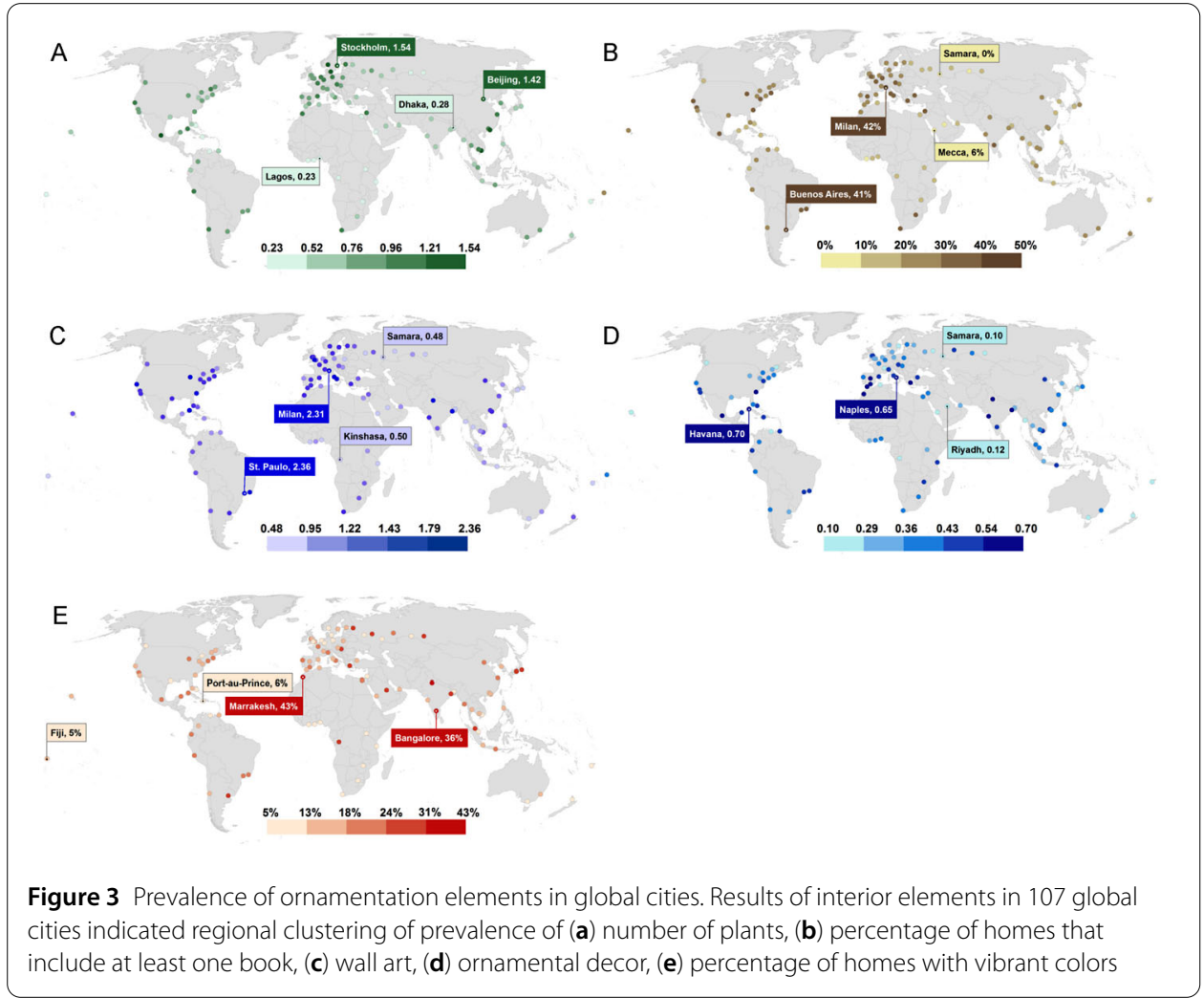

(Fig. 4). Of each element that exhibited significant regional variation, the use of vibrant colors was the least unique to regions, while the presence of wall art was the most unique to regions (Fig. 4). Moran's I results and the ANOVA results aligned to suggest that factors including climate, wealth and culture may statistically influence design in interior spaces. As such, we reject the null hypothesis of randomness of the choice to use plants, books, wall art and vibrant colors to decorate interior spaces across the globe. We do not reject this hypothesis for the use of general decor items, meaning that there is a universal trend of residential ornamentation via artistic objects, but not for more specific elements. We examine these patterns further below.

On the whole, European cities were the most decorated, and cities in Sub-Saharan Africa and Oceania were least decorated (Fig. 4). The top cities for overall ornamentation were found in India, Brazil and Italy (Table 3). In addition, tourist-focused Charleston (U.S.) had high levels of personalization in decor, as did megacities Beijing and Mexico City. Cities with the most austere decorating styles were found in Russia, Saudi Arabia and in SubSaharan Africa (Nigeria, Ghana, Cote d'Ivoire, D.R.C. and Uganda) (Fig. 3). Interestingly, 


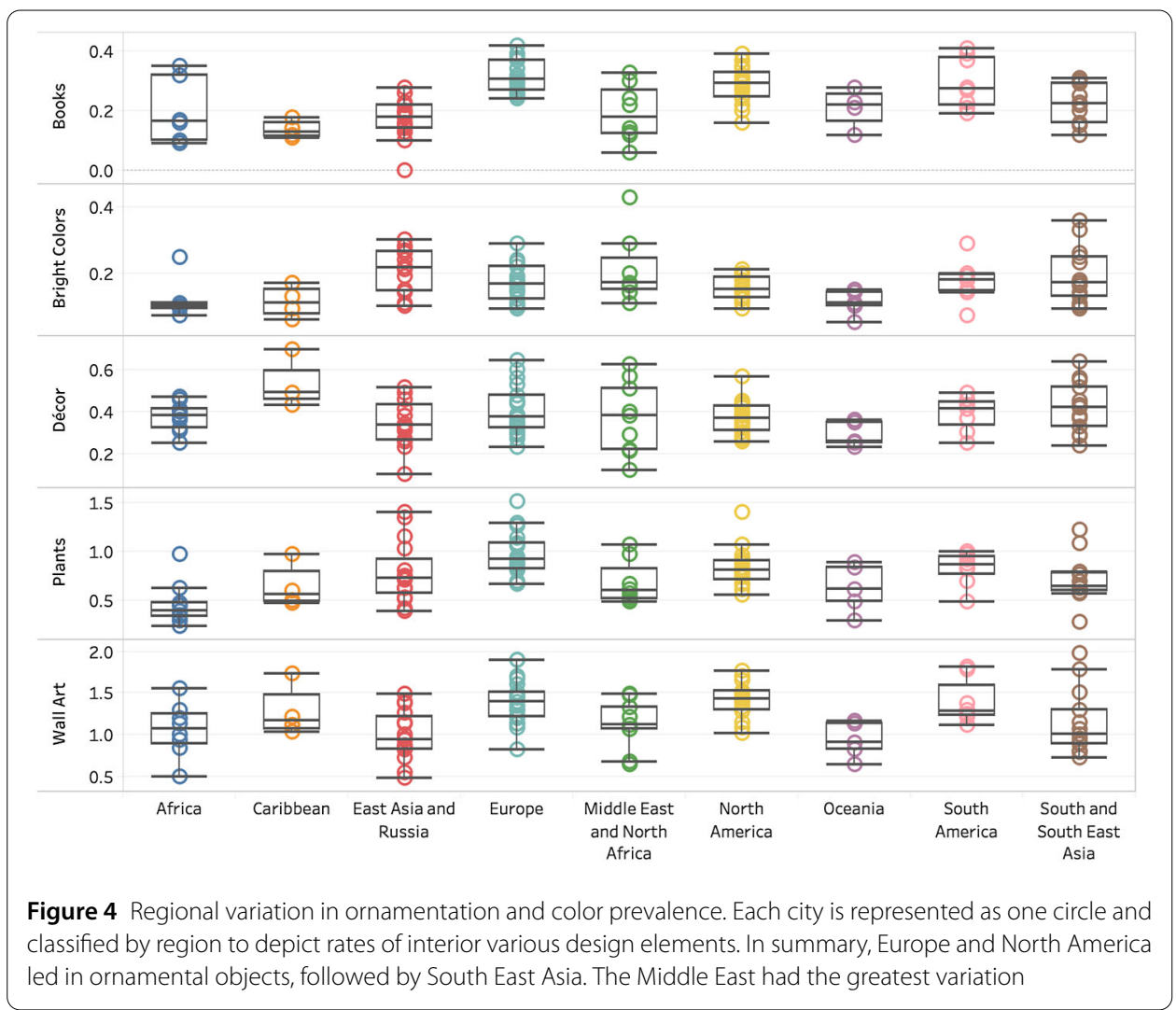

Fiji, a tropical tourist destination, decorated very little, as did Bangladesh, a neighbor to India (Table 3).

\subsubsection{Plants and books}

On average, $46.3 \%$ of listings had plants and $25.5 \%$ of listings had books. We found that Scandinavian and Chinese cities often decorated with plants, where the reverse was true in the Middle East, Africa and Central Russia (Fig. 3(a)). This can be grounded in two veins of reasoning: first, that plants can only survive year-round indoors in colder climates, thus they are invited to live indoors. Second, plants in the tropics may be more likely to harbor insects that could have a negative effect on health. Furthermore, in the Middle East, plants may not be strongly reflected in the culture, or the desert atmosphere may not allow for facile plant maintenance. We found a preponderance of books in the United States, Central/Western Europe, South America, and cities Mexico City and Capetown (Fig. 3(b)). This finding cannot be responsibly tied to literacy rates, as books could be found in other parts of the home (such as the bedroom), but not used as living room ornamentation. However, it may suggest that some cultures may be more interested in sharing books as part of a space for guests while others keep books in more private locations, or do not keep books in the home.

\subsubsection{Art and color}

On average, $62.4 \%$ listings had wall art, $30.2 \%$ had decor and $16.6 \%$ had vibrant colors. Cities in India and Morocco, as well as Madrid, Havana, and Naples had top levels of decor. Qualitative findings support high levels of ornamentation in Morocco, citing the 
Table 3 Top and bottom global cities by presence of decorative elements

\begin{tabular}{|c|c|c|c|c|}
\hline Plants & Books & Wall Art & Decor & Bright Colors \\
\hline \multicolumn{5}{|l|}{ Top Ten Cities } \\
\hline Stockholm, Sweden & Milan, Italy & Delhi, India & Havana, Cuba & $\begin{array}{l}\text { Marrakesh, } \\
\text { Morocco }\end{array}$ \\
\hline Beijing, China & $\begin{array}{l}\text { Buenos Aires, } \\
\text { Argentina }\end{array}$ & Milan, Italy & Naples, Italy & Bangalore, India \\
\hline Mexico City, Mexico & Paris, France & Naples, Italy & Delhi, India & Delhi, India \\
\hline Shenzhen, China & Chicago, U.S. & Sao Paulo, Brazil & Tangier, Morocco & Osaka, Japan \\
\hline Oslo, Norway & Saint Paulo, Brazil & Rio de Janeiro, Brazil & Madrid, Spain & $\begin{array}{l}\text { Riyadh, Saudi } \\
\text { Arabia }\end{array}$ \\
\hline Copenhagen, Denmark & Athens, Greece & Kolkata, India & Marrakesh, Morocco & $\begin{array}{l}\text { Buenos Aires, } \\
\text { Argentina }\end{array}$ \\
\hline Ho Chi Minh, Vietnam & Charleston, U.S. & Charleston, U.S. & Mexico City, Mexico & Athens, Greece \\
\hline Guangzhou, China & Oxford, U.K. & Havana, Cuba & Charleston, U.S. & Kyoto, Japan \\
\hline Berlin, Germany & Rio de Janeiro, Brazil & San Francisco, U.S. & Kolkata, India & $\begin{array}{l}\text { St. Petersburg, } \\
\text { Russia }\end{array}$ \\
\hline Helsinki, Finland & San Francisco, U.S. & Rome, Italy & Granada, Spain & Tokyo, Japan \\
\hline \multicolumn{5}{|l|}{ Bottom Ten Cities } \\
\hline Lagos, Nigeria & Samara, Russia & Samara, Russia & Samara, Russia & Fiji \\
\hline Dhaka, Bangladesh & Mecca, Saudi Arabia & Kinshasa, D.R.C. & Riyadh, Saudi Arabia & Port au Prince, Haiti \\
\hline Apia, Samoa & $\begin{array}{l}\text { Riyadh, Saudi } \\
\text { Arabia }\end{array}$ & Novosibirsk, Russia & Algiers, Algeria & $\begin{array}{l}\text { Abidjan, Cote } \\
\text { d'Ivoire }\end{array}$ \\
\hline Kampala, Uganda & $\begin{array}{l}\text { Abidjan, Cote } \\
\text { d'Ivoire }\end{array}$ & Apia, Samoa & Mecca, Saudi Arabia & Caracas, Venezuela \\
\hline Kinshasa, D.R.C. & Omsk, Russia & Riyadh, Saudi Arabia & Novosibirsk, Russia & Bahamas \\
\hline Dar es Salaam, Tanzania & Accra, Ghana & Mecca, Saudi Arabia & Melbourne, Australia & $\begin{array}{l}\text { Copenhagen, } \\
\text { Denmark }\end{array}$ \\
\hline Omsk, Russia & $\begin{array}{l}\text { Trinidad and } \\
\text { Tobago }\end{array}$ & Kyoto, Japan & Stockholm, Sweden & Harare, Zimbabwe \\
\hline Abidjan, Cote d'Ivoire & Dubai, U.A.E. & Kuala Lumpur, Malaysia & Yangon, Myanmar & Houston, U.S. \\
\hline Kyoto, Japan & Manila, Philippines & Yangon, Myanmar & Caracas, Venezuela & Kampala, Uganda \\
\hline Harare, Zimbabwe & Fiji & Dhaka, Bangladesh & Kinshasa, D.R.C. & Oxford, U.K. \\
\hline
\end{tabular}

"melting pot" of Muslim culture dating back centuries and manifested through vibrant colors [35]. Cities in India, Italy, Brazil and the U.S. had the highest rates of wall art. The distribution of decor was more varied across the globe, but notably present in Southern regions of Africa (Figs. 3(c) and 3(d)).

Conversely, cities in Russia, Saudi Arabia, and Sub-Saharan Africa had very few wall hangings, and were more reserved with their ornamentation decisions. In addition, Kyoto, Kuala Lumpur, Yangon and Dhaka also had low rates of wall art in their homes. These results differentiated Dhaka from neighboring Indian cities such as Delhi and Kolkata, and highlighted Kyoto as a place of potential minimalism, as found with previous results on austerity in Japan [21].

The highest rates of vibrant colors were found in Central Asia, S.E. Asia and North Africa (Fig. 3(e)). The use of calmer colors was found in Sub-Saharan Africa, the Caribbean and Oceania. This distinction seems to have little do to with (warm) climate and light colors' ability to reflect the sun, as S.E. Asia and North Africa have similar temperatures, but prefer to personalize their spaces with saturated walls. Instead, this difference may be attributed to cultural roots. The popularity of bright colors in India, for example, may reflect the decoration styles of the Hindu population, where vibrant oranges are said to be sacred [40]. Ties between Hindu worship and color are key, as every god exhibits a different choice of color; these preferences are manifested in the homes to appease gods like Ganesh, who is said to prefer red and orange [36] (pg. 146). A lack of bright colors may also 
reflect a legacy of cultural heritage. Another property of interest with the use of white and light colors may stem from persistence from colonial ideals of cleanliness [43], persisting into the 21st century.

\subsection{U.S. intra-city level analysis}

With regards to the six U.S. cities of interest, we discovered evidence of weak spatial clustering. Thus, we reject the null hypothesis of randomness in decorative proclivities across geographic space within these cities. We also discovered no statistically significant relationship between decorative behavior and socio-economic indicators within U.S. cities.

First, we found that each element was weakly spatially clustered within each city (Table 4), as denoted by low Moran's I scores, which range from -1.0 to 1.0. Although we can reject the null hypothesis of randomness within cities, the resultant statistics do not communicate a strong conviction towards clustering. For example, elements were very weakly clustered in New York, illustrating that key ornamentation practices, especially decor and bright colors, were not constrained or concentrated in neighborhood culture hearths, but were distributed throughout the city. This finding may re-frame ideas that certain NYC neighborhoods such as Greenwich Village are more 'artsy' than other neighborhoods. Yet, New York neighborhoods also exhibited high z-scores, especially in terms of books and plants. That said, low Moran's I scores may be an artifact of the high sample size of the New York case. Elements in other cities, such as Houston, tended to be slightly more clustered, indicating that ornamentation was not widespread but concentrated in smaller pockets. To contextualize these findings, (Table 5) provides summary statistics of the prevalence and sample size of each city.

While each city cannot be compared to one another, i.e. ranked by Moran's I or z value, these findings suggest that certain elements (such as plants) had stronger clustering tenancies, and that these tenancies were not necessarily equivalent in each city. Still, clustering results of the elements can provide a linkage between the global and intra-city analyses. In

Table 4 Combined Moran's I and z-score statistics for element clustering in selected U.S. cities

\begin{tabular}{llllll}
\hline City/Element & Plants & Books & Wall Art & Decor & Bright Colors \\
\hline Chicago, IL & $0.031(10.42)$ & $0.028(9.39)$ & $0.041(13.64)$ & $0.028(9.42)$ & $0.026(8.84)$ \\
Houston, TX & $0.043(7.18)$ & $0.05(8.36)$ & $0.036(6.06)$ & $0.027(4.62)$ & $0.044(7.35)$ \\
Los Angeles, CA & $0.029(16.74)$ & $0.029(16.66)$ & $0.045(26.4)$ & $0.021(12.34)$ & $0.016(9.42)$ \\
New York, NY & $0.025(43.99)$ & $0.011(19.41)$ & $0.007(11.83)$ & $0.005(9.06)$ & $0.004(7.14)$ \\
Philadelphia, PA & $0.046(12.12)$ & $0.029(7.63)$ & $0.022(5.88)$ & $0.015(4.13)$ & $0.02(5.30)$ \\
Washington, DC & $0.015(3.35)$ & $0.016(3.55)$ & $0.021(4.63)$ & $0.016(3.57)$ & $0.023(5.06)$ \\
\hline
\end{tabular}

Z-scores are in parentheses. All statistical results are found to be weakly clustered with a $p$ value of $<0.001$.

Table 5 Average interior element frequency across selected U.S. cities

\begin{tabular}{lclllll}
\hline City & Number of Listings & Plants & Books & Wall Art & Decor & Bright Colors \\
\hline Chicago, IL & 2772 & 0.84 & 0.36 & 1.54 & 0.40 & 0.19 \\
Houston, TX & 1746 & 0.58 & 0.25 & 1.41 & 0.40 & 0.12 \\
Los Angeles, CA & 9368 & 0.81 & 0.30 & 1.47 & 0.42 & 0.15 \\
New York, NY & 12,537 & 0.76 & 0.34 & 1.41 & 0.36 & 0.17 \\
Philadelphia, PA & 1820 & 0.81 & 0.32 & 1.47 & 0.38 & 0.16 \\
Washington, DC & 4146 & 0.66 & 0.36 & 1.67 & 0.39 & 0.18 \\
Total/Average & 32,389 & 0.76 & 0.33 & 1.48 & 0.39 & 0.16 \\
\hline
\end{tabular}


terms of individual element and color types, plants and wall art were the most localized element and decor was the least localized element (Table 4). Plants were least popular in the low-latitude, warm climate of Houston, TX and most popular in colder cities Chicago and Philadelphia. However, plants were also popular in Los Angeles, which also has a warm climate. Yet, bright colors were less prevalent in Los Angeles and even less so in Houston than other, colder cities, which is consistent with global findings (Table 5).

Neither the linear regression (LR) and geographically weighted regression (GWR) models revealed a consistent correlation between any single or combination of socioeconomic factors and interior space ornamentation. LR and GWR modeled most often yielded $R^{2}$ values of $<0.1$ (See Additional file 1 for model parameters and results). Thus, we accepted the null hypothesis, in this case, that decorative tendencies in these U.S. cities does not depend on the socio-economic characteristics of the surrounding neighborhoods, but instead, appear consistently across the socio-economic spectrum. While a different suite of statistical tests, socio-economic indicators and detected objects may indicate, conversely, a significant relationship between neighborhood social fabric and interior decorative element choice, our particular approach did not. Our results demonstrated that decorating one's living space is not an emblem of a specific demographic profile or extraordinary economic means. In other words, the self-expression and fulfillment inherent to space personalization [2] did not seem to be entirely constrained to privileged groups in large U.S. cities. Of course, it is still easy to believe that those with more disposable income may decorate luxuriously [14], but given today's modern DIY culture [7], it is also possible that the average citizen is going to creative lengths can also decorate their home creatively.

We now visualize two examples of localization of ornamentation elements. The data analysis revealed a difference in local neighborhood use of elements of books and wall art in Philadelphia and New York, respectively (Fig. 5). In Philadelphia, up to 63\% of homes in the neighborhood of Fishtown, known as a gentrifying area, contained books in their living rooms. However, in areas such as Kensington and Point Breeze, the majority of living rooms contained no books (Fig. 5(a)). Smaller living areas in the downtown, Univer-
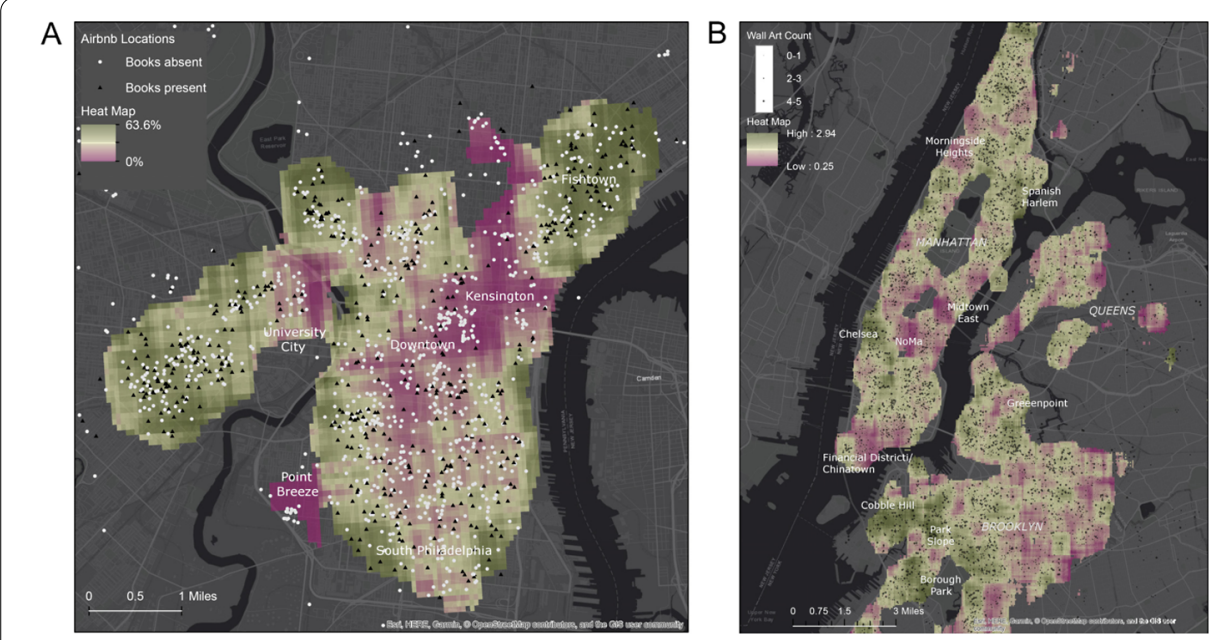

Figure 5 Frequency of ornamental elements by neighborhood. These images illustrate a north-south variation in book presence in Philadelphia, PA where more books are found to the northeast of the downtown area, and wall art frequency in New York City, where key wealthy neighborhoods in Manhattan lack wall art 
sity City and Kensington may contribute to a lack of interior decor such as books. South Philadelphia, an area with a concentrated African American population within the segregated city, had mixed proclivities to display books, and did not differ significantly from other Philadelphia neighborhoods. In New York, units of wall art were concentrated in neighborhoods in Brooklyn, Spanish Harlem and Chelsea which have up to nearly three wall hangings on average (Fig. 5(b)). There is a lack of art, averaging at 0.25 wall hangings per home in North of Manhattan (NoMa), Midtown East and the Financial District, although these areas are home to wealthy Manhattanites. A lack of art and books in Philadelphia and New York may be due to high levels of rental properties and tall apartment buildings that host transient populations and those looking for a more utilitarian living style. Poverty may also be a factor, as lower-income areas of eastern Brooklyn also had fewer units of wall art. Still, the tract-based economic analysis did not point to a correlation between income and prevalence of decorative features.

\section{Discussion and conclusions}

\subsection{Discussion}

In our study of geolocated images of interior living spaces, we discovered that residents in world regions tend to decorate in statistically-different ways regarding wall art, color, plants, and books - but exhibit similarities in their use of general decor. At the U.S. intracity level, the wide distribution of ornamentation across different socioeconomic neighborhoods confirmed that carefully personalizing spaces is not reserved for any certain geographic or social group. As such, although home values and income differ widely across cities, we find a commonality in intentions to cultivate a thoughtful interior space. This care and self-expression could be seen as a unifying, not dividing, statistic for even the most segregated cities.

These findings are part of a novel set of studies that take advantage of online datasets that evidence agent-based decisions and personal practices (i.e. decorating one's own home) to help define culture and culture hearths using quantitative analytics (e.g. [20]). These findings can also be situated within a larger family of geospatial urban data on human behavior that augment traditional top-down demographics. More broadly, this analysis illustrates how geographical cultural practices can be found within large online datasets, and assessed using advancements in computer vision.

\subsection{Limitations}

The conclusions drawn from this research are limited in part by potential biases inherent to the data source. First, it is unclear how well the data hosts represent the larger population. Hosts who list their properties are willing and able to rent extra space in their homes, and thus, may not represent the typical resident who does not have extra space or is not interested in hosting visitors. This may include residents with small children, strict adherence to religious observances, illness, or other reasons. A study of Airbnb rental hosts revealed that hosts are motivated by financial reasons, but also to cultivate social and interpersonal interactions $[55,56]$. In addition, the typical host may be different depending on the type of listing. In a study of Airbnb rentals in London, it was shown that room rentals were associated with highly-educated expat renters, while entire homes were found in high-end neighborhoods [10].

Next, due to the competitiveness for bookings, decorating listings in order to appeal to consumers is a strategy that may compromise how an owner would naturally decorate 
his or her home if it was not posted to the public and furnished to entice visitors. Design factors are key marketing tools in the hospitality industry [57-59], where stylish spaces and comfort are key elements of travelers' choices of where to stay [60-62], and the servicescape is no exception [3]. It is also clear that hosts seek guidance when decorating. An online search retrieves many advice articles, including excerpts from the Washington Post [63] and the Airbnb official blog [64], for decorating one's home as a short-term rental. Informally, these suggest that the owner limits displaying many pictures of family, and perhaps choose local art and photos to add a 'sense of place'. In a typical home that is not for rent, there may be more personalized items, such as family portraits, children's drawings, religious icons or memorials, or even brighter colors. Moreover, hosts may be influenced to emulate design factors from existing successful site listings (and even peruse customer feedback for hints on what visitors might like). While the extent to which this process drives decorative decision-making for hosts is unknown, we acknowledge that it adds potential noise to photos as indicators of individual self-expression.

Finally, our examination of urban areas provided a perspective that may not be found in rural, small city, or even suburban areas, which tend to be more traditional [8]. Urban areas tend to have more modern spaces that can lack traditional symbolic elements that are more prevalent in rural locales [4]. In the future, including geolocatable residential interior images from sites such as Craigslist or U.S.-focused Zillow [14], may help add diversity to the image corpus and analysis.

Future directions for this research include training our model to discover different styles of artwork, perhaps ranging by time period, medium and cultural origin. We can also train our model to discover the proliferation of meaningful elements such as historical emblems, world flags, photos of world leaders, modern technological devices, etc. As such, we can help bridge the gap between big data and human geographical, cultural and urban theory to learn more about widespread practices and patterns around the world. We can also test new hypotheses generated and informed through qualitative cultural research on a larger, more expansive sample of cases.

\section{Additional material}

Additional file 1: Supplementary information. (PDF 6.9 MB)

Additional file 2: Multi-page .csv of all 107 cities and element counts. Table of all global cities with average rates of decor prevalence for each indicator. (CSV 7 kB)

\section{Acknowledgements}

The authors acknowledge Hanzhou Chen and Luca Maria Aiello for helpful conversations, and the availability of the online Airbnb API for providing the data used in this study. The authors also acknowledge the support of NVIDIA Corporation with a donation of the Titan Xp GPU used for this research.

\section{Funding}

The authors declare no funding sources for this work.

\section{Abbreviations}

ACS, American Community Survey; ANOVA, Analysis of Variance; API, Application Programming Interface; GIS, Geographic Information Systems; GWR, Geographically-Weighted Regression; HSV, Hue, Saturation, Value; LR, Linear Regression; NAS, Neural Architecture Search; RCNN, Recurrent Convolutional Neural Network. 


\section{Competing interests}

The authors declare that they have no competing interests.

\section{Authors' contributions}

CA conceived of the study. $X \mathrm{~L}, \mathrm{CA}$ and $\mathrm{SR}$ designed the study. $\mathrm{XL}$ and $\mathrm{SR}$ retrieved and cleaned the data. $\mathrm{XL}, \mathrm{CA}, \mathrm{ZH}$ and SR analyzed data. XL, CA and ZH designed visualizations and XL and CA wrote the paper. All authors read and approved the final manuscript.

\section{Author details}

'Department of Geography, The Pennsylvania State University, University Park, USA. ²Department of Geography, University of Utah, Salt Lake City, USA. ${ }^{3}$ Department of Architecture, The Pennsylvania State University, University Park, USA.

\section{Publisher's Note}

Springer Nature remains neutral with regard to jurisdictional claims in published maps and institutional affiliations.

Received: 16 August 2018 Accepted: 24 January 2019 Published online: 08 February 2019

\section{References}

1. Lennon SJ, Fairhurst A, Peatross F (1991) Apparel and furniture attribute importance as a function of self-monitoring. Fam Consum Sci Res J 19(4):292-302

2. Malnar JM, Vodvarka F (1992) The interior dimension: a theoretical approach to enclosed space. Reinhold, New York

3. Lin IY (2004) Evaluating a servicescape: the effect of cognition and emotion. Int J Contemp Hosp Manag 23(2):163-178

4. Sayrafi M (2015) The modern Palestinian home. In: Asher JA, Blossom N (eds) Handbook of interior design. Wiley, Chinchester, pp 260-272

5. Bonnes M, Giuliani MV, Amoni F, Bernard Y (1987) Cross-cultural rules for the optimization of the living room. Environ Behav 19(2):204-227

6. Snodin M, Howard M (1996) Ornament: a social history since 1450. Yale University Press, New Haven

7. Clarke A (2002) The aesthetics of social aspiration. In: Miller D (ed) Home possessions. Berg Publishers, Oxford, pp 23-45

8. Mercer C (2014) Middle class construction: domestic architecture, aesthetics and anxieties in Tanzania. J Mod Afr Stud 52(2):227-250

9. Countryman CC, Jang S (2006) The effects of atmospheric elements on customer impression: the case of hotel lobbies. Int J Contemp Hosp Manag 18(7):534-545

10. Quattrone G, Greatorex A, Quercia D, Capra L, Musolesi M (2018) Analyzing and predicting the spatial penetration of airbnb in us cities. EPJ Data Sci 7(1):31

11. Wilson MA, Mackenzie NE (2000) Social attributions based on domestic interiors. J Environ Psychol 20(4):343-354

12. Ordonez V, Jagadeesh V, Di W, Bhardwaj A, Piramuthu R (2014) Furniture-geek: understanding fine-grained furniture attributes from freely associated text and tags. In: Applications of computer vision (WACV), 2014 IEEE winter conference on pp 317-324. IEEE

13. Karayev S, Trentacoste M, Han H, Agarwala A, Darrell T, Hertzmann A, Winnemoeller H (2014) Recognizing image style. In: Proceedings of the British machine vision conference. BMVA Press. https://doi.org/10.5244/C.28.122

14. Poursaeed O, Matera T, Belongie S (2018) Vision-based real estate price estimation. Mach Vis Appl 29(4):667-676

15. Zhang M, Luo L (2018) Can user generated content predict restaurant survival: deep learning of yelp photos and reviews. Social Science Research Network (SSRN). https://doi.org/10.2139/ssrn.3108288

16. Santani D, Gatica-Perez D (2015) Loud and trendy: crowdsourcing impressions of social ambiance in popular indoor urban places. In: Proceedings of the 23rd ACM international conference on multimedia. ACM, New York, pp 211-220

17. Gebru T, Krause J, Wang Y, Chen D, Deng J, Aiden EL, Fei-Fei L (2017) Using deep learning and Google street view to estimate the demographic makeup of neighborhoods across the United States. Proc Natl Acad Sci USA 114:13108-13113

18. Honig CD, MacDowall L (2017) Spatio-temporal mapping of street art using Instagram. First Monday 22(3)

19. Wang J, Korayem M, Crandall D (2013) Observing the natural world with Flickr. In: Proceedings of the IEEE international conference on computer vision workshops, pp 452-459

20. Nguyen LS, Ruiz-Correa S, Mast MS, Gatica-Perez D (2018) Check out this place: inferring ambiance from airbnb photos. IEEE Trans Multimed 20(6):1499-1511

21. Rahimi S, Liu X, Andris C (2016) Hidden style in the city: an analysis of geolocated airbnb rental images in ten major cities. In: Proceedings of the 2nd ACM SIGSPATIAL workshop on smart cities and urban analytics. ACM, New York, p 7

22. Oliver P (2007) Built to meet needs: cultural issues in vernacular architecture. Architectural Press, Oxford

23. Crook CJ (1997) The dimensionality of stratification-related cultural practices in Australia. Aust NZ J Sociol 33(2):226-238

24. Evans MD, Kelley J, Sikora J, Treiman DJ (2010) Family scholarly culture and educational success: books and schooling in 27 nations. Res Soc Stratif Mobil 28(2):171-197

25. De Graaf PM (1986) The impact of financial and cultural resources on educational attainment in the Netherlands. Sociol Educ 59(4):237-246

26. Evans MDR, Kelley J, Sikora J (2014) Scholarly culture and academic performance in 42 nations. Soc Forces 92(4):1573-1605

27. Ray A, Margaret W (2002) Programme for international student assessment (PISA) 2000 technical report. Organisation for Economic Co-Operation and Development (OECD) Publishing. https://www.oecd.org/pisa/data/33688233.pdf

28. Flannery MC (2005) Jellyfish on the ceiling and deer in the den: the biology of interior decoration. Leonardo 38(3):239-244 
29. Ulrich RS (1991) Effects of interior design on wellness: theory and recent scientific research. J Health Care Inter Des 3(1):97-109

30. Dijkstra K, Pieterse ME, Pruyn A (2008) Stress-reducing effects of indoor plants in the built healthcare environment: the mediating role of perceived attractiveness. Prev Med 47(3):279-283

31. Lohr VI, Pearsons-Mims CH, Goodwin GK (1996) Interior plants may improve worker productivity and reduce stress in a windowless environment. J Environ Hortic 14(2):97-100

32. Raanaas RK, Evensen KH, Rich D, Sjøstrøm G, Patil G (2011) Benefits of indoor plants on attention capacity in an office setting. J Environ Psychol 31(1):99-105

33. Ames KL (1980) Material culture as nonverbal communication: a historical case study. J Am Cult 3(4):619-641

34. Lawrence RJ (1987) What makes a house a home? Environ Behav 19(2):154-168

35. McCloud AB (1996) "This is a Muslim home" signs of difference in the African-American row house. In: Metcalf BD (ed) Making muslim space in North America and Europe, vol 22. University of California Press, Berkeley, pp 65-73

36. Sahney P (2012) Religion and houses in a transnational setting: a study of interior decoration of Hindu immigrants from India in the United States. Doctoral dissertation, Indiana University

37. Aslam MM (2006) Are you selling the right colour? A cross-cultural review of colour as a marketing cue. J Mark Commun 12(1):15-30

38. Wise BK, Wise JA (1988) Nasa technical report: the human factors of color in environmental design: a critical review. https://ntrs.nasa.gov/search.jsp?R=19890006161

39. Eysenck HJ (1941) A critical and experimental study of colour preferences. Am J Psychol 54(3):385-394

40. Madden TJ, Hewett K, Roth MS (2000) Managing images in different cultures: a cross-national study of color meanings and preferences. J Int Mark 8(4):90-107

41. Adams FM, Osgood CE (1973) A cross-cultural study of the affective meanings of color. J Cross-Cult Psychol $4(2): 135-156$

42. Park N-K, Pae JY, Meneely J (2010) Cultural preferences in hotel guestroom lighting design. J Inter Design 36(1):21-34

43. Jacobs JM, Cairns S (2008) The modern touch: interior design and modernisation in post-independence Singapore. Environ Plan A 40(3):572-595

44. Lenclos J-P, Lenclos D (2004) Colors of the world: the geography of color. Norton, New York

45. Zagorski M (2007) The geography of significant colorants: antiquity to the twentieth century. Master's thesis, George Mason University

46. Gou A (2013) Method of urban color plan based on spatial configuration. Color Res Appl 38(1):65-72

47. Abadi M, Agarwal A, Barham P, Brevdo E, Chen Z, Citro C, Corrado GS, Davis A, Dean J, Devin M, Ghemawat S, Goodfellow I, Harp A, Irving G, Isard M, Jia Y, Jozefowicz R, Kaiser L, Kudlur M, Levenberg J, Mané D, Monga R, Moore S, Murray D, Olah C, Schuster M, Shlens J, Steiner B, Sutskever I, Talwar K, Tucker P, Vanhoucke V, Vasudevan V, Viégas F, Vinyals O, Warden P, Wattenberg M, Wicke M, Yu Y, Zheng X (2015) TensorFlow: large-scale machine learning on heterogeneous systems. Software available from tensorflow.org. https://www.tensorflow.org/

48. Szegedy C, Vanhoucke V, loffe S, Shlens J, Wojna Z (2016) Rethinking the inception architecture for computer vision. In: Proceedings of the IEEE conference on computer vision and pattern recognition, pp 2818-2826

49. Deng J, Dong W, Socher R, Li L-J, Li K, Fei-Fei L (2009) ImageNet: a large-scale hierarchical image database. In: CVPR09

50. Huang J, Rathod V, Sun C, Zhu M, Korattikara A, Fathi A, Fischer I, Wojna Z, Song Y, Guadarrama S, Murphy K (2017) Speed/accuracy trade-offs for modern convolutional object detectors. In: The IEEE conference on computer vision and pattern recognition. CVPR

51. Ren S, He K, Girshick R, Sun J (2015) Faster r-cnn: towards real-time object detection with region proposal networks. In: Advances in neural information processing systems (NIPS). Neural Information Processing Systems Foundation, Inc., La Jolla, pp 91-99

52. Zoph B, Vasudevan V, Shlens J, Le QV (2017) Learning transferable architectures for scalable image recognition. arXiv preprint. arXiv:1707.07012

53. Lin T-Y, Maire M, Belongie S, Hays J, Perona P, Ramanan D, Dollár P, Zitnick CL (2014) Microsoft coco: common objects in context. In: European conference on computer vision. Springer, Berlin, pp 740-755

54. Farrell RJ, Booth JM (1975) Design handbook for imagery interpretation equipment. Technical report, Boeing Aerospace Company, Seattle, Washington

55. Lampinen A, Cheshire C (2016) Hosting via airbnb: motivations and financial assurances in monetized network hospitality. In: Proceedings of the 2016 CHI conference on human factors in computing systems. ACM, New York, pp 1669-1680

56. Quattrone G, Proserpio D, Quercia D, Capra L, Musolesi M (2016) Who benefits from the sharing economy of airbnb? In: Proceedings of the 25th international conference on world wide web, pp 1385-1394. International World Wide Web Conferences Steering Committee

57. Booms BH, Bitner MJ (1982) Marketing services by managing the environment. Cornell Hotel Restaur Adm Q 23(1):35-40

58. Rutkin KM (2005) User preference of interior design elements in hotel lobby spaces. Master's thesis, University of Florida

59. Mattila AS, Wirtz J (2001) Congruency of scent and music as a driver of in-store evaluations and behavior. J Retail $77(2): 273-289$

60. Han H, Hsu L-TJ, Lee J-S (2009) Empirical investigation of the roles of attitudes toward green behaviors, overall image, gender, and age in hotel customers' eco-friendly decision-making process. Int J Contemp Hosp Manag 28(4):519-528

61. Becker EJ (2009) The proximity hotel: a case study on guest satisfaction of sustainable luxury environments. Master's thesis, The University of North Carolina at Greensboro

62. Fidzani LC, Caughey C (2015) First impressions of hotel lobbies as inferences about hotels: a marketing tool. Botsw J Bus 7(1):1-12

63. Kiefer E Setting up an airbnb? Here are the basics, the extras and the no-nos. AirBnb Blog (14 August, 2018). https://www.washingtonpost.com/lifestyle/home/setting-up-an-airbnb-here-are-the-basics-the-extras-and-theno-nos/2018/08/13/53fbb492-998b-11e8-843b-36e177f3081c_story.html

64. AirBNB (2014) Attract more guests: 10 simple tips from home staging expert Meridith Baer. AirBnb Blog (17 April, 2014). https://blog.atairbnb.com/attract-guests-10-simple-tips-home-staging-expert-meridith-baer/ 